\title{
Power-Efficient Relay Selection in Cooperative Networks Using Decentralized Distributed Space-Time Block Coding
}

\author{
Lu Zhang and Leonard J. Cimini Jr. \\ Department of Electrical and Computer Engineering, University of Delaware, Newark, DE 19716, USA \\ Correspondence should be addressed to Lu Zhang, luzhang@udel.edu
}

Received 1 May 2007; Accepted 8 September 2007

Recommended by R. K. Mallik

Distributed space-time block coding (Dis-STBC) achieves diversity through cooperative transmission among geographically dispersed nodes. In this paper, we present a power-efficient relay-selection strategy for decentralized Dis-STBC in a selective decodeand-forward cooperative network. In particular, for a two-stage network, each decoded node broadcasts a small amount of information with limited power. This node then utilizes its own and its neighbors' information to decide whether or not to act as a relay for the source information. In this way, only part of the decoded set will act as relays. Further, by applying the idea of this relay-selection strategy to each relaying hop in a multihop network, a power-efficient hop-by-hop routing strategy is formulated. The outage analyses and simulations are presented to illustrate the advantage of these strategies.

Copyright (C) 2008 L. Zhang and L. J. Cimini Jr.. This is an open access article distributed under the Creative Commons Attribution License, which permits unrestricted use, distribution, and reproduction in any medium, provided the original work is properly cited.

\section{INTRODUCTION}

Cooperative diversity is a set of techniques that exploits the spatial diversity available among a collection of distributed single-antenna terminals (e.g., see [1]). In most proposed cooperative systems, a two-stage relaying strategy is used. In the first stage, a source transmits and all the other nodes listen; in the second stage, the relays cooperate to retransmit the source message to the destination. Several relay management strategies can be employed. In selective decode-andforward relaying, a node is called a decoded node if it can correctly decode the source message; then some subset of the decoded nodes is selected to act as the relay set. In [2], a distributed space-time block code (Dis-STBC) was proposed in which each relay transmits one unique column of the underlying STBC matrix. So that each relay knows which column to transmit, most of the proposed Dis-STBC schemes [2-8] require a central control unit or full internode negotiations.

Several decentralized Dis-STBC schemes have been proposed to implement code assignment at the relays without control signaling (e.g., see $[9,10])$. In decentralized DisSTBC, one possible relay-selection strategy is for all the nodes in the decoded set to act as relays for the source information; we call this the all-select approach. For decentralized
Dis-STBC, it has been observed [11] that, when the number of relays is much greater than the number of columns in the underlying STBC matrix, any further increase in the number of relays, although consuming more power, will not result in additional diversity benefit. Obviously, then, such a strategy, where all the nodes in the decoded set retransmit the source message, might be wasteful of power, especially when the number of decoded nodes is large.

In this paper, we propose a more power-efficient relayselection strategy for decentralized Dis-STBC. In the proposed relay-selection strategy, each decoded node broadcasts a small amount of information with limited power, then utilizes its own and its local neighbors' information to decide whether to act as a relay or not. Based on this information, only a subset of the nodes in the decoded set will act as relays. In order to incur the least overhead while also being robust when a deep fade occurs over some internode channels, we do not require that each decoded node can correctly receive the information from all of the other decoded nodes. In particular, as an example, we focus on $m$-group Dis-STBC [9], in which each relay randomly and independently chooses one column from the underlying STBC matrix.

The paper is organized as follows. The system model for a two-stage network is described in Section 2. In Section 3, 
using $m$-group Dis-STBC as an example, we propose a power-efficient relay-selection strategy for decentralized DisSTBC. In Section 4, an asymptotic upper bound on the outage for the $m$-group Dis-STBC is derived, and the powerefficiency advantage of the proposed relay-selection strategy is illustrated. Simulation results in a two-stage network are given in Section 5. In Section 6, by extending the idea of the proposed relay-selection strategy and also using $m$ group Dis-STBC as an example, a power-efficient hop-byhop routing strategy is formulated for a multihop network that uses decentralized Dis-STBC at each relaying hop. Finally, Section 7 provides concluding remarks.

\section{SYSTEM MODEL}

We assume a two-stage protocol that uses a selective decodeand-forward relaying strategy, as illustrated in Figure 1. In particular, we consider a network with $M$ single-antenna nodes. When one source-destination pair, $(s, d)$, is active, all the remaining $M-2$ nodes can serve as potential relays. Define the decoded set as the set of $N(N \leq M-2)$ nodes that can correctly decode the transmitted signal from the source. Note that the decoded set is random, varying with the instantaneous channel gains. $K(K \leq N)$ decoded nodes are then selected to relay the source message. We assume that nodes cannot transmit and receive simultaneously. In addition, we assume perfect synchronization and a quasi-static propagation environment.

When using the all-select relay-selection strategy, $K=N$; however, this decentralized relay-selection strategy might be inefficient. Each relay must also determine which column of the code matrix to transmit. Generally, the code assignment among the relays requires a central control unit or full internode negotiations. Several decentralized Dis-STBC schemes have been proposed to implement code assignment without control signaling (e.g., see $[9,10])$. In this paper, we will focus on $m$-group Dis-STBC [9] which is described next.

In $m$-group Dis-STBC, each relay independently chooses one column at random out of the $L$ columns in the underlying STBC matrix. Specifically, denote $S$ as the underlying $L$ column STBC matrix, where the row of $\mathbf{S}$ indicates the time index and the column indicates the transmit antenna index. When $\mathbf{S}$ has $m$ columns (i.e., $L=m$ ), it is equivalent to dividing the relays into $m$ groups, where the relays within a certain group choose the same column. However, since some groups might be empty, this scheme does not ensure the maximum possible diversity order, $L$. In particular, the number of distinct columns randomly selected by the $K(K \leq N)$ relays is denoted as $V(1 \leq V \leq L)$. Then, denote $B_{v}(v=1, \ldots, V)$ as the $v$ th subset of the set of $K$ relays, and $K_{v}$ as the number of relays in $B_{v}$. The relays within $B_{v}$ will transmit the $v$ th column out of the $V$ randomly selected distinct columns.

This scheme is a special case of the randomized DisSTBC proposed in [10]. Let $\mathbf{R}=\left[\mathbf{r}_{1}, \mathbf{r}_{2}, \ldots, \mathbf{r}_{K}\right]$ represent the randomized matrix with $L$ rows and $K$ columns, where $\mathbf{r}_{j}=\left[r_{1, j}, \ldots, r_{L, j}\right]^{T}$ is the randomized coefficient vector independently generated by relay $j(j=1, \ldots, K)$. In the $m$ group scheme, the random coefficients $r_{i, j}$ are drawn from the discrete-element set $\{0,1\}$. In this case, $\mathbf{r}_{j}$ belongs to

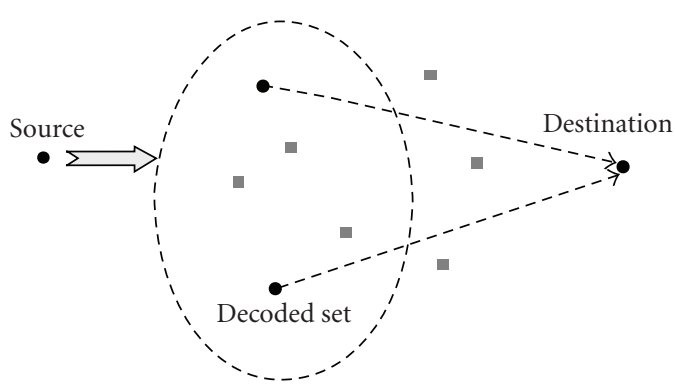

Figure 1: A two-stage selective decode-and-forward cooperative network.

$\left\{\mathbf{d}_{i}, i=1, \ldots, L\right\}$, where $\mathbf{d}_{i}$ is the vector of all zeros except for the $i$ th position which is 1 . Let the instantaneous channel coefficients $\alpha_{i, j}$ capture the effects of path loss and flat Rayleigh fading between node $i$ and node $j$. Denot $\boldsymbol{\alpha}=$ $\left[\alpha_{1, d}, \ldots, \alpha_{K, d}\right]^{T}$ as the channel coefficient vector for transmissions from the $K$ relays to the destination, and $\mathbf{Z}$ as additive white Gaussian noise with the variance $N_{0}{ }^{1}$ per complex dimension. Further, let $\beta_{i, d}=r_{i, 1} \alpha_{1, d}+\cdots+r_{i, K} \alpha_{K, d}(i=$ $1, \ldots, L)$ represent the equivalent instantaneous channel coefficients and $\boldsymbol{\beta}=\left[\beta_{1, d}, \ldots, \beta_{L, d}\right]^{T}$. Then, the received signal $\mathrm{Y}$ at the destination is

$$
\mathrm{Y}=\mathrm{SR} \boldsymbol{\alpha}+\mathrm{Z}=\mathrm{S} \boldsymbol{\beta}+\mathrm{Z}
$$

Based on (1), by estimating the equivalent channel coefficients $\boldsymbol{\beta}$, the conventional coherent detection algorithm of STBC can still be used for randomized Dis-STBC.

\section{POWER-EFFICIENT RELAY-SELECTION STRATEGY}

For decentralized Dis-STBC, the all-select relay-selection strategy might result in a substantial waste of power. If only a subset of the decoded nodes is selected to act as relays, good performance might be achieved with much less power consumption in the second stage.

In the $m$-group scheme, the number of randomly selected distinct columns $V(1 \leq V \leq L)$ determines the achieved diversity benefit. If the random column selection is performed after the relay selection, only selecting part of the $N$ decoded nodes as relays might result in a decrease in $V$ (i.e., a decrease in the diversity gain). Thus, the challenge is to effectively select a subset of the decoded nodes as relays to try to maintain the same diversity gain as the all-select strategy, while introducing sufficiently small overhead. In what follows, by using power-limited one-way control signals, a power-efficient relay-selection strategy is presented for the $m$-group Dis-STBC. We call this strategy local- $k$-best relay selection, and it works as follows.

(i) Each decoded node randomly chooses a column so that $V(1 \leq V \leq L)$ becomes the number of distinct columns randomly selected by the $N$ decoded nodes.

\footnotetext{
${ }^{1}$ Without loss of generality, $N_{0}$ is normalized to 1 .
} 
(ii) Each decoded node broadcasts the local mean power gain of the channel from itself to the destination and the index of its randomly selected column, by using a low transmit powe $P_{\mathrm{bc}}$. The broadcast transmissions by all the decoded nodes use a multichannel CSMA MAC protocol [12], which provides "soft" channel reservation by combining CSMA with CDMA, for example. By using this MAC protocol, the control signaling is one-way traffic (no response is required for broadcasting). The effect of the hidden-node problem could also be reduced well.

(iii) At each decoded node, if it finds that there exist at least $k(k \geq 1)$ neighbors which have the same selected column as itself and which have larger local mean power gain than itself, then this decoded node will not act as a relay.

In this strategy, for any particular random selection of columns by the $N$ decoded nodes (i.e., for any given value of $V$ ), each of the $V$ randomly selected distinct columns will be transmitted by at least one relay such that $V \leq K \leq N$. Thus, when only a subset of all the decoded nodes is selected as relays, the achieved diversity gain is the same as using the all-select strategy. In addition, since the $K$ selected relays have relatively larger local mean power gains, better performance will be achieved.

The power overhead of the local- $k$-best strategy is $N P_{\mathrm{bc}}$. In order to incur the least possible overhead and also to be robust when a deep fade occurs over some internode channels, we do not require each decoded node to correctly receive the information from all of the other decoded nodes. With a low transmit power for the broadcast signal, the neighbors of each decoded node will only be a subset of the decoded set. Since the amount of the local information required to be broadcasted by each decoded node is quite small, by further using a multichannel CSMA MAC protocol [12] for broadcastings by all the decoded nodes, the resulting time overhead should be negligible when compared with the time used for the transmission of data packets.

\section{PERFORMANCE ANALYSIS}

In this section, we illustrate the power-efficiency advantage of the local- $k$-best strategy by deriving an asymptotic upper bound on the outage probability for the $m$-group Dis-STBC.

\subsection{Asymptotic upper bound on the outage for m-group}

Assume that the all-select or local- $k$-best strategy is used such that $K(V \leq K \leq N)$ decoded nodes are selected as relays. A two-stage transmission is in outage if the receive SNR at the destination is below a given SNR threshold $\eta_{t}$. The outage probability at the destination is denoted as $p_{\text {out }, d}$. Denote $P_{s}$ as the transmit power of the source node and $P_{r}$ as the transmit power of each relay. (When coding is used and the code rate is not equal to one, $P_{s}$ and $P_{r}$ represent the power per information symbol.) Denote the mean values of the channel power gains $\left|\alpha_{s, d}\right|^{2}$ and $\left|\alpha_{j, d}\right|^{2}$ as $\mu_{s, d}$ and $\mu_{j, d}(j=1, \ldots, K)$, respectively. Further, denote $\mu_{\min , v}$ as the minimum value among $\mu_{j, d}, j \in B_{v}(v=1, \ldots, V)$. Next, an asymptotic upper bound on $p_{\text {out }, d}$ is obtained.

Theorem 1. For any given decoded set and particular random column selection by all of the decoded nodes, an asymptotic upper bound on $p_{\text {out }, d}$ for the m-group Dis-STBC is given by

$$
p_{\text {out }, d} \leq \frac{\eta_{t}^{V+1} /(V+1) !}{P_{s} \mu_{s, d} \times P_{r}^{V} \times\left(\mu_{\min , 1} \times \cdots \times \mu_{\min , V}\right)} .
$$

This asymptotic upper bound is tight when $P_{s} \mu_{s, d}$ and $P_{r} \mu_{\min , v}$ are sufficiently large for all $v \in\{1, \ldots, V\}$.

Proof. For any particular random column selection by the $N$ decoded nodes, the nonzero equivalent channel coefficients $\beta_{v, d}(v=1, \ldots, V)$ can be expressed as

$$
\beta_{v, d}=\sum_{j \in B_{v}} \alpha_{j, d} \quad(v=1, \ldots, V)
$$

Based on (3), it can be seen that the $\beta_{1, d}, \ldots, \beta_{V, d}$ are independent complex Gaussian variables since there is no intersection among $B_{v}(v=1, \ldots, V)$. Thus, the power gains of the nonzero equivalent channels $\left|\beta_{v, d}\right|^{2}(v=1, \ldots, V)$ are independent exponential random variables with means $\sum_{j \in B_{\nu}} \mu_{j, d}$. By applying the conventional coherent detection algorithm for STBC [13] and combining the received signals from the two stages, the outage probability at the destination $p_{\text {out }, d}$ is

$$
p_{\text {out }, d}=\operatorname{Pr}\left\{\left(P_{s}\left|\alpha_{s, d}\right|^{2}+\sum_{v=1}^{V} P_{r}\left|\beta_{v, d}\right|^{2}\right) \leq \eta_{t}\right\} .
$$

It can be seen that, for a particular random column selection by any given $N$ decoded nodes, the $m$-group scheme is equivalent to formulating $V(1 \leq V \leq L)$ "virtual relays," each of which transmits one distinct column out of the $V$ selected columns. Here, the equivalent channel coefficients between the virtual relays $v$ and the destination are $\beta_{v, d}(v=1, \ldots, V)$, which are independent complex Gaussian variables. Thus, it can be viewed as applying the centralized Dis-STBC [2] with a $V$-column code matrix to the $V$ "virtual relays." By exploiting the results in [14] for the outage analysis of centralized Dis-STBC, for any given decoded set and particular random column selection by all the decoded nodes, we can express the upper bound on $p_{\text {out }, d}$ for the $m$-group Dis-STBC as

$$
p_{\text {out }, d} \leq \frac{\eta_{t}^{V+1} /(V+1) !}{P_{s} \mu_{s, d} \times P_{r} \sum_{j \in B_{1}} \mu_{j, d} \times \cdots \times P_{r} \sum_{j \in B_{V}} \mu_{j, d}} .
$$

This upper bound is tight when $P_{s} \mu_{s, d}$ and $P_{r} \sum_{j \in B_{v}} \mu_{j, d}$ are sufficiently large for all $v$. Clearly, $\mu_{\min , v} \leq \sum_{j \in B_{v}} \mu_{j, d}(v=$ $1, \ldots, V)$. Thus, based on (5), we obtain the asymptotic upper bound as given in (2). 


\subsection{Advantage of local- $k$-best}

With the given total power consumption in a two-stage transmission, the asymptotic upper bound on $p_{\text {out }, d}$ can be optimized by using the local- $k$-best strategy when the values of $P_{\mathrm{bc}}$ and $k(k \geq 1)$ are properly chosen such that $K<N$. This is shown in the following theorem.

Theorem 2. With a given source power $P_{s}$ and a given power consumption in the second stage $P_{2}$, for the m-group Dis-STBC, the asymptotic upper bound on $p_{\mathrm{out}, d}$ when using the local- $k$ best strategy is smaller than or equal to that when using the all-select strategy.

Proof. With a given power consumption $P_{2}$ in the second stage, we have $P_{r}=P_{2} / \mathrm{N}$ for the all-select strategy and $P_{r}=P_{2} / K(V \leq K \leq N)$ for the local- $k$-best strategy. For any given decoded set and particular random column selection by the $N$ decoded nodes, denote $D_{v}(v=1, \ldots, V)$ as the $v$ th subset of the decoded set. The randomly selected column by the decoded nodes in $D_{v}$ is the $v$ th column out of the $V$ randomly selected distinct columns. Obviously, $B_{v} \subseteq D_{v}$. Further, denote $\varepsilon_{\min , v}$ as the minimum value among $\mu_{j, d}, j \in$ $D_{v}(v=1, \ldots, V)$. Clearly, when the all-select strategy is used, $K=N$ and $B_{v}=D_{v}$ so that $\mu_{\min , v}=\varepsilon_{\min , v}$. Since (2) is obtained for general $K(V \leq K \leq N)$ and $B_{v}\left(B_{v} \subseteq D_{v}\right)$, according to (2), we get

all-select:

$$
p_{\text {out }, d} \leq \frac{\eta_{t}^{V+1} /(V+1) !}{P_{s} \mu_{s, d} P_{2}^{V}(1 / N)^{V}\left(\varepsilon_{\min , 1} \times \cdots \times \varepsilon_{\min , V}\right)},
$$

local- $k$-best:

$$
p_{\text {out }, d} \leq \frac{\eta_{t}^{V+1} /(V+1) !}{P_{s} \mu_{s, d} P_{2}^{V}(1 / K)^{V}\left(\mu_{\min , 1} \times \cdots \times \mu_{\min , V}\right)} .
$$

When the local- $k$-best strategy is used but some inappropriate values are set up for $P_{\mathrm{bc}}$ and $k(k \geq 1)$ such that $K=N$, we have $B_{v}=D_{v}$ so that $\mu_{\min , v}=\varepsilon_{\min , v}$ for all $v$. In this case, the local- $k$-best strategy is equivalent to the all-select strategy; in particular, this might result when $P_{\mathrm{bc}}$ is very small or when $k$ is large.

The values of $P_{\mathrm{bc}}$ and $k(k \geq 1)$ could be properly chosen such that $K<N$ (the optimal values of $P_{\mathrm{bc}}$ and $k$ will be investigated by simulations). In this case, $B_{v} \subset D_{v}$ for at least one $v \in\{1, \ldots, V\}$. As we know, the local- $k$-best strategy is designed to select $K_{v}$ decoded nodes from $D_{v}$ to act as relays $(v=1, \ldots, V)$, and it also tries to choose the $K_{v}$ relays that have larger local mean power gains when compared with the other decoded nodes in $D_{v}$. For any $v$ with $B_{v} \subset D_{v}$, in the worst case, the $K_{v}$ selected relays include the "poorest" decoded node in $D_{v}$ (i.e., the node having the smallest local mean power gain among all the decoded nodes in $D_{v}$ ) so that $\mu_{\min , v}=\varepsilon_{\min , v}$. This situation might happen when the "poorest" decoded node in $D_{v}$ has no neighbors or all of its neighboring decoded nodes choose different columns from itself. In the other situations, clearly, $\mu_{\min , v}>\varepsilon_{\min , v}$. Thus, when $K<N$, we have $\mu_{\min , v} \geq \varepsilon_{\min , v}(v=1, \ldots, V)$. According to (6) and (7), when $K<N$, the asymptotic upper bound on $p_{\text {out }, d}$ with the local- $k$-best strategy is smaller than that with the all-select strategy.

\subsection{Key parameters in the local-k-best strategy}

Based on the discussion in the previous subsection, $P_{\mathrm{bc}}$ and $k$ are the two key parameters in the local- $k$-best strategy. $P_{\mathrm{bc}}$ is the power used by each decoded node to broadcast its local information. If one decoded node finds that there exist at least $k(k \geq 1)$ neighbors which are better relay candidates than itself, it will not act as a relay. The value of $P_{\mathrm{bc}}$ will affect the number of neighbors for each decoded node and, subsequently, affect the number of relays $K(V \leq K \leq N)$. If $P_{\mathrm{bc}}$ is large, the power overhead might be too large. On the other hand, if $P_{\mathrm{bc}}$ is too small, the number of neighbors of each decoded node might be zero so that $K=N$. In the next section, we will use simulations to investigate the effect on performance for different values of $P_{\mathrm{bc}}$ to obtain an appropriate range of values.

With an increase in $k(k \geq 1)$, at each decoded node the possibility that there exist at least $k$ neighbors which are better relay candidates decreases; then, the number of relays $K$ increases. This will result in an increase in power consumption in the second stage. However, for the $m$-group scheme with the local- $k$-best strategy, whatever the value of $k$ is, all $V(1 \leq V \leq L)$ distinct columns which are randomly selected by all $N$ decoded nodes will be transmitted by $K(V \leq K \leq N)$ relays. That is to say, an increase in $k$ will not provide more diversity benefit. Intuitively, when $k$ is smaller, the power efficiency is better. In the next section, we will use simulations to show the effect on performance when varying $k$.

\section{SIMULATION RESULTS}

In this section, under realistic propagation conditions, including the effects of path loss and flat Rayleigh fading, the outage performance of the $m$-group Dis-STBC is evaluated with different relay-selection strategies, including the local$k$-best and all-select strategies.

\subsection{Simulation environment}

We consider a square coverage area with diagonal dimension $d_{\max }$ and $M$ uniformly distributed single-antenna halfduplex nodes. To implement power allocation in a decentralized way, it is assumed that constant transmit power $P_{t}$ is used for each node, that is, $P_{s}=P_{r}=P_{t} .{ }^{2}$ Thus, for the all-select strategy, the total power to transmit one message is $P=P_{s}+N P_{r}=(1+N) P_{t}$; for the local- $k$-best $(k \geq 1)$ strategy, the power overhead resulting from broadcasting local information is included in the performance evaluation such that the total power to transmit one message is $P=$ $P_{s}+K P_{r}+N P_{\mathrm{bc}}=(1+K) P_{t}+N P_{\mathrm{bc}}$. Here, the time overhead

\footnotetext{
2 Two ad hoc, yet more efficient, power allocation strategies are suggested in [15] for decentralized Dis-STBC.
} 
resulting from broadcasting local information is not considered since it could be negligible when compared with the time used for transmitting data packets.

The outage probability of the farthest $(s, d)$ pair is evaluated. To determine the SNR threshold $\eta_{t}$, we follow a similar argument as in [16]; that is, $\eta_{t}$ is determined as $b \times\left(2^{2 r}-\right.$ 1) for two-stage cooperative transmission. The parameter $r$ (bps/Hz) is the achieved spectral efficiency of the noncooperative direct transmission. The parameter $b$ ranges from 1 to about 6.4, depending on the degree of used coding [17].

To evaluate the performance in a more realistic environment, the wireless channels include the effects of path loss and flat Rayleigh fading. In addition, the geographic distributions of the potential relays are random. The outage probability is obtained by averaging over node locations and Rayleigh fadings. As in [16], the powers are normalized by $P_{\max }$ which is the transmit power required, for the maximal possible separation of source and destination $d_{\max }$, to achieve a given spectral efficiency $r$ in direct transmission without shadow fading and Rayleigh fading. The outage curves are plotted as a function of the normalized average power $P_{\mathrm{av}}$, which is the average consumed power per two-stage transmission.

\subsection{Outage probability}

Here, we use the parameter $\xi=P_{\mathrm{bc}} / P_{\max }$ to investigate the effect on outage performance when varying $P_{\mathrm{bc}}$. This is shown in Figure 2 for the two-group scheme with local-one-best strategy when there are $M=16$ nodes in the network and $L=2$ columns in the STBC matrix. In particular, we use an Alamouti code [18]. It can be observed that a ratio $\xi$ in the interval $[0.09,0.11]$ achieves the optimal performance. Similarly, when $M=16$ and $L=2$, the optimal $\xi$, $\xi_{\text {opt }}$, is around 0.1 for the two-group scheme with local-two-best strategy. In addition, when $M=32$ and $L=2$, the $\xi_{\text {opt }}$ is around 0.05 for the two-group scheme with local- $k$-best $(k=1,2)$ strategy. As an empirical result, $\xi_{\text {opt }}$ is approximately equal to $1 /(M-2)$. Recall that $M-2$ is the number of all potential relays in the network; thus, $M-2$ is also the maximum possible number of the decoded nodes.

With the empirically optimal value for $P_{\mathrm{bc}} / P_{\max }$, the outage performance of the $m$-group scheme with local- $k$-best strategy is investigated when varying $k$. Simulation results are shown in Figure 3 for the two-group scheme with the local$k$-best $(k=1,2)$ strategy and the all-select strategy, when $M=16$ nodes and $L=2$ using an Alamouti code. Clearly, it can be seen that, even with the overhead included, the localone-best strategy is much more power-efficient than the allselect strategy. In particular, a $2 \mathrm{~dB}$ advantage can be observed at an outage probability of $10^{-2}$. When $P_{\mathrm{av}}$ is large, the local-two-best strategy is also more power-efficient than the all-select strategy. Obviously, the advantage of the local$k$-best strategy decreases with an increase in $k$. This is because an increase in $k$ will not provide additional diversity benefit but will result in an increased power consumption in the second stage.

Results are shown in Figure 4 when $M=32$. Clearly, it can be seen that, as the number of nodes $M$ increases,

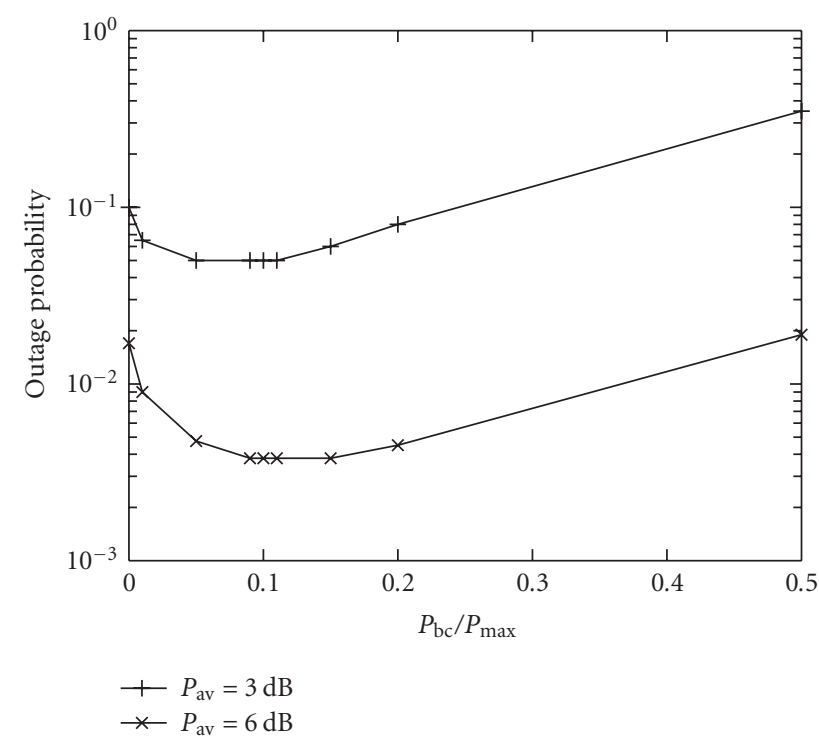

Figure 2: Outage probability as a function of $P_{\mathrm{bc}} / P_{\max }$ for the twogroup scheme with the local-one-best strategy with $M=16, L=2$ $(r=2 \mathrm{bps} / \mathrm{Hz})$.

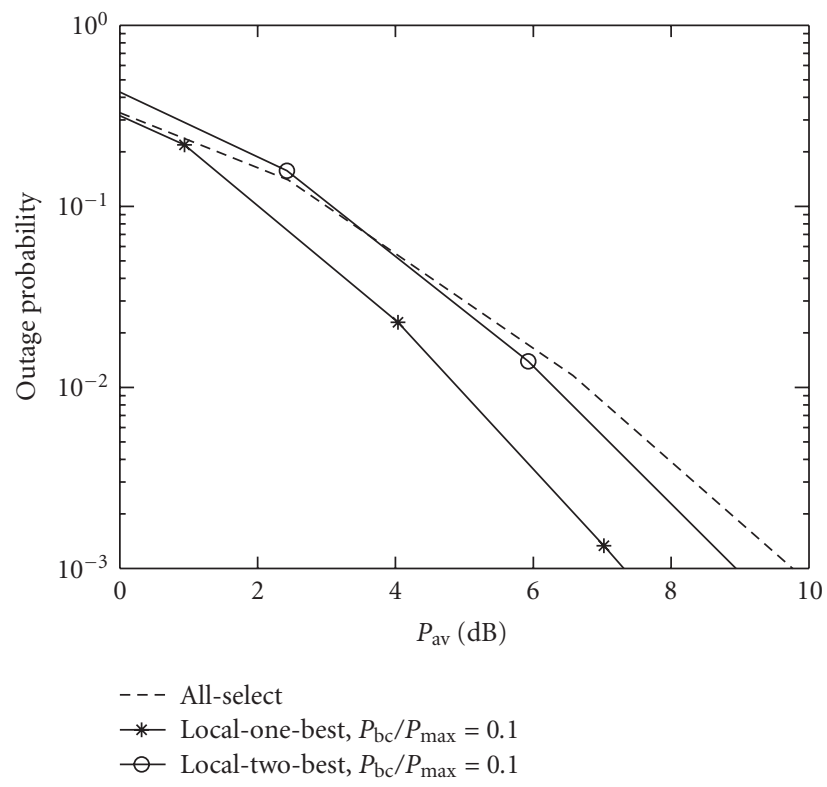

FIGURE 3: Outage probability as a function of the total transmission power of the two stages, $P_{\mathrm{av}}$, for the two-group scheme with $M=16$, $L=2(r=2 \mathrm{bps} / \mathrm{Hz})$.

the performance gap between the all-select strategy and the local- $k$-best $(k=1,2)$ strategy becomes larger. In this case, the local-one-best strategy is almost $3 \mathrm{~dB}$ better than the allselect strategy at an outage probability of $10^{-2}$. With a given transmit power for the source, on average, the number of decoded nodes will increase with an increase in the number of total nodes, $M$. Thus, when $M$ increases, the all-select strategy will waste more power in the second stage to achieve the required performance at the destination. 


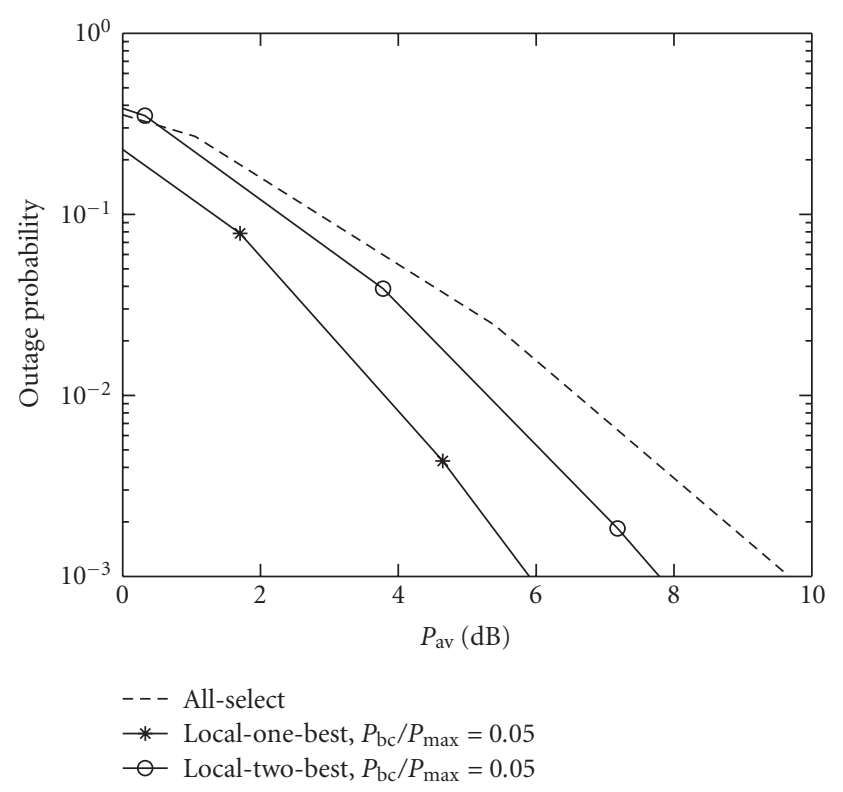

FIgURE 4: Outage probability as a function of the total transmission power of the two stages, $P_{\mathrm{av}}$, for the two-group scheme with $M=32$, $L=2(r=2 \mathrm{bps} / \mathrm{Hz})$.

\section{EXTENSION TO MULTIHOP NETWORK}

There has been growing interest in applying Dis-STBC to a multihop wireless network to achieve cooperative diversity by using a virtual antenna array at each relaying hop [19-23]. In these works, it has been shown that this type of ST-coded cooperative routing has much better performance than traditional node-by-node single-relay routing. However, just as for a two-stage network, in most of these works, for a multihop network, the implementation of Dis-STBC at each relaying hop requires a central control unit or full internode negotiations so that every selected relay knows which column of the underlying STBC matrix to transmit. Obviously, this could require significant overhead. In this section, we will investigate applying decentralized Dis-STBC to a multihop network. In particular, by extending the idea of the power-efficient relay-selection strategy in a two-stage network and also using $m$-group Dis-STBC as an example, a power-efficient routing strategy will be proposed for a multihop network that uses decentralized Dis-STBC at each relaying hop. In the multihop case, since each relay might have multiple local mean power gains to the multiple receiving nodes, some modification must be done when utilizing the local mean power gain information at relays.

In a decode-and-forward multihop network, since a successful end-to-end transmission requires the source message to be correctly decoded by some node(s) at each hop, the destination will be in outage if any one certain hop is in outage. Thus, the end-to-end outage performance is determined by the outage performance of each single hop. In particular, we consider a $J$-hop $(J>2)$ network. If we denote $p_{\text {out }, n}$ as the outage probability at hop $n(n=0, \ldots, J-1)$ and $p_{\text {out }, d}$ as the outage probability at the destination, then we have

$$
p_{\text {out }, d}=1-\prod_{n=0}^{J-1}\left(1-p_{\text {out }, n}\right) \approx \sum_{n=0}^{J-1} p_{\text {out }, n} .
$$

In the decentralized scenario, it is difficult to obtain global channel information. Thus, it is desirable to design a hopby-hop routing strategy which optimize $p_{\text {out }, d}$ by optimizing $p_{\text {out }, n}$ for every $n \in\{0, \ldots, J-1\}$.

When designing a hop-by-hop routing strategy for a multihop network that uses selective decode-and-forward relaying, the relay selection at each relaying hop is the key to the design. Since we could optimize the performance independently for each single hop, the power-efficient relay-selection strategy in a two-stage network can be naturally applied to each relaying hop with appropriate modification. Then, the power efficiency of the routing can be improved. Note that, in this paper, a routing strategy just means a path-selection strategy; it is not a real routing protocol.

\subsection{Multihop network system model}

We consider a $J$-hop $(J>2)$ network that uses a selective decode-and-forward relaying strategy, as illustrated in Figure 5. The $J-1$ node sets $S_{1}, \ldots, S_{J-1}$ are located from the source to the destination. The source is denoted as $S_{0}$ and the destination is denoted as $S_{J}$. The $W_{n}$ nodes in $S_{n}(n=$ $1, \ldots, J-1)$ are potential forwarding relays at relaying hop $n$. Here, as an example, it is assumed that the node sets $S_{n}(n=1, \ldots, J-1)$ are formulated through a destinationinitiated power-limited flooding. As in a two-stage network, we assume that the instantaneous channel between any two single-antenna half-duplex nodes captures the effects of path loss and flat Rayleigh fading. In addition, we assume perfect synchronization and a quasi-static environment. Finally, we assume that the receiving nodes at each hop can only utilize the transmission in the current hop to make a decision.

At relaying hop $n(n=1, \ldots, J-1)$, the transmitting node set is $S_{n}$; the decoded set within $S_{n}$ is defined as the set of $N_{n}\left(N_{n} \leq W_{n}\right)$ decoded nodes that can correctly decode the transmission from hop $n-1$. Note that the decoded sets are random, varying with the instantaneous channel gains. At relaying hop $n(n=1, \ldots, J-1), K_{n}\left(K_{n} \leq N_{n}\right)$ decoded nodes are selected to relay the source message. In particular, when $m$-group Dis-STBC is used at each relaying hop, the number of distinct columns randomly selected by the $N_{n}$ decoded nodes in $S_{n}$ is denoted as $V_{n}\left(1 \leq V_{n} \leq L\right)$. Then, denote $B_{n, v}\left(v=1, \ldots, V_{n}\right)$ as the $v$ th subset of the set of $K_{n}\left(K_{n} \leq N_{n}\right)$ selected relays, and $K_{n, v}$ as the number of relays in $B_{n, v}$. The relays within $B_{n, v}$ will transmit the $v$ th column out of the $V_{n}$ randomly selected distinct columns.

\subsection{Power-efficient hop-by-hop routing strategy}

When $m$-group Dis-STBC is used, the all-select relayselection strategy can be used at each relaying hop. In this case, at relaying hop $n(n=1, \ldots, J-1)$, all $N_{n}$ decoded nodes in the transmitting node set $S_{n}$ forward the source 


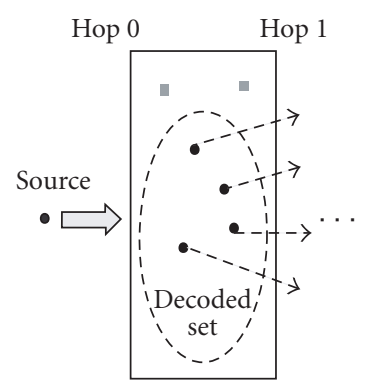

$S_{1}$

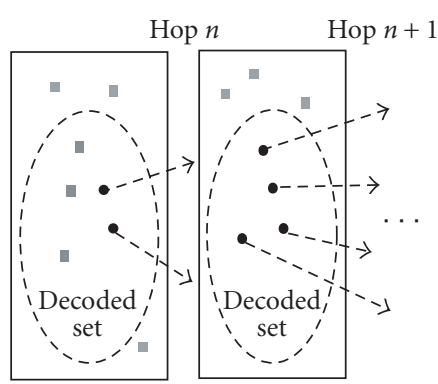

$S_{n}$

$S_{n+1}$

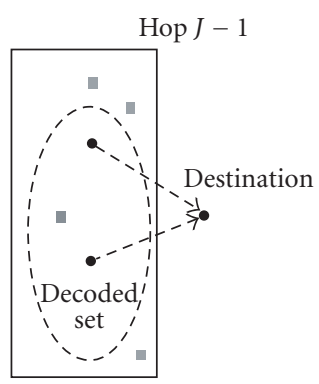

$S_{J-1}$

FIGURE 5: A J-hop selective decode-and-forward cooperative network.

message. We call this all-select routing. This routing strategy might result in a substantial waste of power, similar to the all-select relay-selection strategy in a two-stage network.

If the local- $k$-best $(k \geq 1)$ relay-selection strategy is used at each relaying hop, a power-efficient hop-by-hop routing strategy might be formulated. However, in the multihop case, each relay in $S_{n}(n=1, \ldots, J-1)$ might have multiple local mean power gains to the $W_{n+1}\left(W_{n+1} \geq 1\right)$ receiving nodes in $S_{n+1}$. Thus, we cannot directly use the local- $k$-best $(k \geq 1)$ relay selection. Intuitively, a good measurement of the channel power gain at each relay in $S_{n}$ is an average over its local mean power gains to the $W_{n+1}$ receiving nodes in $S_{n+1}$. Here, we choose the geometric average and denote this as the locally averaged mean power gain to the next node set. Then, the local- $k$-best relay-selection strategy for a two-stage network can be simply modified by letting each decoded node in $S_{n}(n=1, \ldots, J-1)$ broadcast its locally averaged mean power gain to $S_{n+1}$, instead of broadcasting its local mean power gain to the destination. By applying the modified local- $k$-best $(k \geq 1)$ relay-selection strategy to each relaying hop, a power-efficient hop-by-hop local- $k$-best routing is formulated.

When using the local- $k$-best routing strategy, the achieved diversity gain at each relaying hop is the same as using the all-select routing strategy; however, less power is used to relay the source message. In addition, at relaying hop $n(n=1, \ldots, J-1)$, since the $K_{n}\left(V_{n} \leq K_{n} \leq N_{n}\right)$ selected relays have relatively larger locally averaged mean power gains to the receiving node set $S_{n+1}$, better performance will be achieved.

\subsection{Performance analysis}

Based on (8), the end-to-end outage performance $p_{\text {out, } d}$ is determined by the outage probability at each hop. In this section, we illustrate the power-efficiency advantage of the local- $k$-best routing strategy by deriving an asymptotic upper bound on the outage probability at relaying hop $n(n=$ $1, \ldots, J-1)$.

"Relaying hop $n$ is in outage" means that all nodes within $S_{n+1}$ cannot correctly decode the source message forwarded by the $K_{n}$ selected relays within $S_{n}$. Denote $P_{t}$ as the transmit power of each node. At relaying hop $n(n=1, \ldots, J-1)$, denote $\mu_{i, j}$ as the mean value of the channel power gain from the selected relay $i$ in $S_{n}$ to node $j$ in $S_{n+1}\left(i=1, \ldots, K_{n}\right.$, $\left.j=1, \ldots, W_{n+1}\right)$. Further, denote $g_{\min , v}$ as the minimum value among $\prod_{j \in S_{n+1}} \mu_{i, j}, i \in B_{n, v}\left(v=1, \ldots, V_{n}\right)$. Next, an asymptotic upper bound on $p_{\text {out }, n}(n=1, \ldots, J-1)$ is obtained.

Theorem 3. When $m$-group Dis-STBC is used at relaying hop $n(n=1, \ldots, J-1)$, for any given decoded set in $S_{n}$ and particular random column selection by the $N_{n}$ decoded nodes, an asymptotic upper bound on $p_{\mathrm{out}, n}$ is given by

$$
p_{\text {out }, n} \leq \frac{\eta_{t}^{V_{n} W_{n+1}} /\left(V_{n} !\right)^{W_{n+1}}}{P_{t}^{V_{n} W_{n+1}} \times g_{\min , 1} \times \cdots \times g_{\min , V_{n}}} .
$$

This asymptotic upper bound is tight when $P_{t}^{W_{n+1}} g_{\min , v}$ is sufficiently large for all $v \in\left\{1, \ldots, V_{n}\right\}$.

The proof of Theorem 3 can be done through the quite similar way used in the proof of Theorem 1; thus, it is omitted for the sake of brevity.

With a given power consumption at relaying hop $n$, the asymptotic upper bound on $p_{\text {out }, n}$ can be optimized by using the local- $k$-best routing strategy when the values of $P_{\mathrm{bc}}$ and $k(k \geq 1)$ are properly chosen such that $K_{n}<N_{n}$. This is shown in the following theorem.

Theorem 4. With a given power consumption $P_{n}$ for the transmission at relaying hop $n(n=1, \ldots, J-1)$, when $m$-group Dis-STBC is used at relaying hop $n$, the asymptotic upper bound on $p_{\text {out }, n}$ when using the local- $k$-best routing strategy is smaller than or equal to that when using the all-select routing strategy.

The proof of Theorem 4 can be done through the quite similar way used in the proof of Theorem 2; thus, it is omitted for the sake of brevity.

Since $p_{\text {out }, n}$ for each $n \in\{1, \ldots, J-1\}$ can be improved by using the local- $k$-best routing strategy, based on (8), the end-to-end outage performance $p_{\text {out }, d}$ can be improved.

\subsection{Simulation results}

In this subsection, under realistic propagation conditions, including the effects of path loss and flat Rayleigh fading, the end-to-end outage performance is evaluated with different 
routing strategies, including the local- $k$-best routing and allselect routing strategies.

As for a two-stage network, we consider a square coverage area with diagonal dimension $d_{\max }$ and $M$ uniformly distributed single-antenna half-duplex nodes. The $x$ - and $y$ coordinates of all nodes are i.i.d. uniformly distributed random variables on the interval $\left[0, d_{\max } / \sqrt{2}\right]$. Denote $\operatorname{dist}\{i, j\}$ as the distance between node $i$ and node $j$. In simulations, when using destination-initiated power-limited flooding to form the node sets $S_{n}(n=1, \ldots, J-1)$, we simply use $d_{\text {inthop }}$ to represent the reliable coverage range resulting from a limited flooding power. For every particular geographic distribution of the $M$ nodes, the node sets for a given $(s, d)$ pair in a $J$-hop $(J>2)$ network are formulated as

$$
\begin{aligned}
S_{J} & =\{\text { destination }\}, \\
S_{J-1} & =\left\{i \mid \operatorname{dist}\{i, \text { destination }\} \leq d_{\text {inthop }}\right\}, \\
S_{J-2} & =\left\{i \mid \operatorname{dist}\{i, j\} \leq d_{\text {inthop }}, \quad j \in S_{J-1}, i \notin S_{J} \cup S_{J-1}\right\}, \\
\ldots & \ldots \\
S_{n-1} & =\left\{i \mid \operatorname{dist}\{i, j\} \leq d_{\text {inthop }}, \quad j \in S_{n}, i \notin S_{J} \cup S_{J-1} \cup \cdots \cup S_{n}\right\},
\end{aligned}
$$$$
\text { ....... }
$$

The processing stops when the source is found such that $S_{0}=\{$ source $\}$. In simulations, for a given $(s, d)$ pair, the geographic distributions of all the other $M-2$ potential relays are randomly generated and a large number of realizations are considered. Thus, $J$ is a dynamic value for a given $(s, d)$ pair and particular $d_{\text {inthop. }}$. Define $J_{\text {av }}$ as the average hop number where the average is taken over all considered realizations of random geographic distributions.

In particular, we evaluate the end-to-end outage performance for the $(s, d)$ pair with source $=\left(0,0.5 d_{\max } / \sqrt{2}\right)$ and destination $=\left(d_{\max } / \sqrt{2}, 0.5 d_{\max } / \sqrt{2}\right)$. It is assumed that the constant transmit power $P_{t}$ is used for each node. Thus, the total power to transmit one message over $J$ hops is $P=\sum_{n=0 \sim J-1} P_{n}$, where $P_{n}$ is the power consumption at hop $n(n=0, \ldots, J-1)$. For both routing strategies, $P_{0}=P_{t}$. For the all-select routing strategy, $P_{n}=N_{n} P_{t}(n=$ $1, \ldots, J-1)$; for the local- $k$-best routing strategy, the power overhead resulting from broadcasting local information is included in the performance evaluation such that $P_{n}=K_{n} P_{t}+$ $N_{n} P_{\text {bc }}(n=1, \ldots, J-1)$. Similar to Section 5.1, in a $J$-hop $(J>2)$ network, the SNR threshold $\eta_{t}$ is determined as $b \times\left(2^{J r}-1\right)$ since the $J$-hop cooperative transmission has a $1: J$ bandwidth penalty compared to the direct transmission. The powers are normalized by $P_{\max }$. The definitions of $r, b$, and $P_{\max }$ are the same as in Section 5.1. The outage curves are plotted as a function of the normalized average power $P_{\mathrm{av}}$, which is the average consumed power per $J$-hop transmission.

As in a two-stage network, here, we also use the parameter $\xi=P_{\mathrm{bc}} / P_{\max }$ to investigate the effect of varying the broadcast power $P_{\mathrm{bc}}$ on the end-to-end outage performance. This is shown in Figure 6 for the local-one-best routing when there are $M=100$ nodes in the network, $d_{\text {inthop }} / d_{\max }=1 / 6$, and $L=2$ columns in the underlying STBC matrix of the $m$-group Dis-STBC. It can be observed that a ratio $\xi$ in the

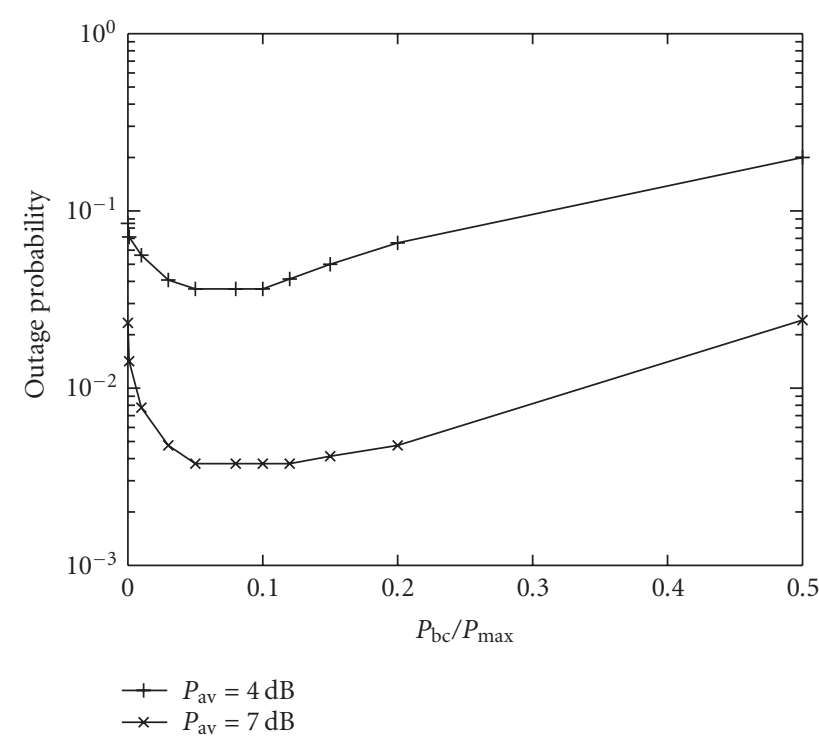

Figure 6: Outage probability as a function of $P_{\mathrm{bc}} / P_{\max }$ for the local-one-best routing using the two-group scheme with $M=100$, $d_{\text {inthop }} / d_{\max }=1 / 6, J_{\mathrm{av}} \approx 5.11, L=2(r=2 \mathrm{bps} / \mathrm{Hz})$.

interval $[0.05,0.1]$ achieves the optimal performance. Similarly, $\xi_{\text {opt }}$ is in the interval $[0.05,0.1]$ for the local-two-best routing when $M=100, d_{\text {inthop }} / d_{\max }=1 / 6$, and $L=2$. In these simulations, $J_{\mathrm{av}} \approx 5.11$. Then, on average, the number of the relaying node sets is $J_{\mathrm{av}}-1$. Thus, on average, the maximum possible number of the decoded nodes per relaying hop is $(M-2) /\left(J_{\mathrm{av}}-1\right)$. As an empirical result, $\xi_{\mathrm{opt}}$ is approximately equal to $1 /\left[(M-2) /\left(J_{\mathrm{av}}-1\right)\right]$.

According to the obtained range of values for $\xi_{\mathrm{opt}}$, with choosing $P_{\mathrm{bc}} / P_{\max }=0.08$ and using the $m$-group Dis-STBC, the end-to-end outage performance of the local- $k$-best routing is investigated when varying $k$. Simulation results are shown in Figure 7 for the local- $k$-best $(k=1,2)$ routing and all-select routing when $M=100$ nodes, $d_{\text {inthop }} / d_{\max }=$ $1 / 6$, and $L=2$ using an Alamouti code. Clearly, it can be seen that, even with the overhead included, the local-onebest routing is much more power-efficient than the all-select routing. In particular, a $2.5 \mathrm{~dB}$ advantage can be observed at an outage probability of $10^{-2}$. As in a two-stage network using local- $k$-best relay selection, the advantage of the local- $k$ best routing decreases with an increase in $k$.

\section{CONCLUSIONS AND FUTURE WORKS}

In this paper, for a two-stage network that uses selective decode-and-forward relaying, we presented a power-efficient relay-selection strategy for a particular decentralized DisSTBC scheme ( $m$-group). The power-efficiency advantage of the proposed local- $k$-best $(k \geq 1)$ relay-selection strategy was illustrated through the outage analysis. Under realistic propagation conditions, including the effects of path loss and flat Rayleigh fading, we evaluated the outage performance of the $m$-group scheme with different relay-selection strategies. It was found that, when compared with the allselect relay-selection strategy, the local- $k$-best relay-selection 


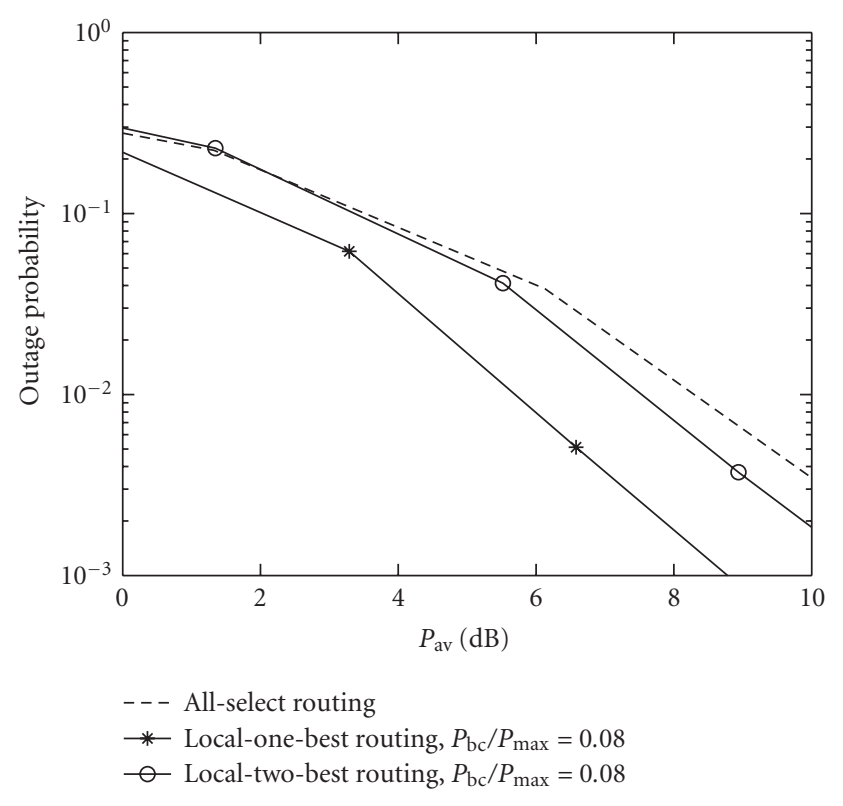

FIGURE 7: Outage probability as a function of the total transmission power of the $J$ hops, $P_{\mathrm{av}}$, for the two-group scheme with $M=100$, $d_{\text {inthop }} / d_{\max }=1 / 6, J_{\mathrm{av}} \approx 5.11, L=2(r=2 \mathrm{bps} / \mathrm{Hz})$.

strategy is much more power-efficient even with the additional power overhead included. In addition, by using the modified local- $k$-best relay-selection strategy at each relaying hop, a power-efficient hop-by-hop routing strategy was proposed for a multihop, selective, decode-and-forward network that uses the $m$-group Dis-STBC at each relaying hop. Under realistic propagation conditions, the end-to-end outage performance was evaluated for different routing strategies. It was found that, when compared with the all-select routing strategy, the local- $k$-best routing strategy is much more power-efficient even with the additional power overhead included. Although, in this paper, the local- $k$-best $(k \geq 1)$ relay-selection/routing strategies were presented by using the $m$-group Dis-STBC as an example, these strategies can be naturally extended to other decentralized Dis-STBC schemes (such as the continuous randomized scheme [10]).

To implement the local- $k$-best strategies, the implementation for all decoded nodes to broadcast local information is important. In a cooperative network with half-duplex limitation of nodes, since we try to implement the broadcastings of decoded nodes with incurring small overhead, the oneway control traffic is preferred. In addition, the local- $k$-best strategies would like to let the broadcasting by each decoded node reach all neighboring decoded nodes. Based on the considerations described above, when using a random access protocol to implement the broadcastings by all the decoded nodes, we would not like to utilize CSMA combined with the RTS/CTS mechanism. Instead, we currently consider using a multichannel CSMA MAC protocol [12]. This protocol combines CSMA with CDMA, for example; it reduces the effect of the hidden-node problem elegantly and is quite suitable for the scenario where the broadcastings are intended to reach all neighbors. Of course, other approaches will also be investigated. Furthermore, in the multihop case, the distribution for the local mean power gains of all interhop channels might be another implementation issue worthy of concern. It is advisable to combine this information distribution into the process of forming and maintaining relay clusters (i.e., node sets defined in this paper). Besides the destinationinitiated power-limited flooding scheme used for simulations in this paper, so many other proposed (distributed) clustering schemes could be explored in further research. In the future, by paying more attention to these implementation issues, we will try to implement local- $k$-best strategies in practical communication protocols for wireless cooperative networks.

\section{ACKNOWLEDGMENTS}

This material is based on research sponsored by the Air Force Research Laboratory, under Agreement no. FA9550-06-10077. The US government is authorized to reproduce and distribute reprints for governmental purposes notwithstanding any copyright notation thereon.

\section{REFERENCES}

[1] J. N. Laneman, D. N. C. Tse, and G. W. Wornell, "Cooperative diversity in wireless networks: efficient protocols and outage behavior," IEEE Transactions on Information Theory, vol. 50, no. 12, pp. 3062-3080, 2004.

[2] J. N. Laneman and G. W. Wornell, "Distributed space-timecoded protocols for exploiting cooperative diversity in wireless networks," IEEE Transactions on Information Theory, vol. 49, no. 10, pp. 2415-2525, 2003.

[3] S. Barbarossa and G. Scutari, "Distributed space-time coding for multihop networks," in Proceedings of the IEEE International Conference on Communications (ICC '04), vol. 2, pp. 916-920, Paris, France, June 2004.

[4] S. Barbarossa and G. Scutari, "Distributed space-time coding for regenerative relay networks," IEEE Transactions on Wireless Communications, vol. 4, no. 5, pp. 2387-2399, 2005.

[5] H. T. Cheng, H. Mheidat, M. Uysal, and T. M. Lok, "Distributed space-time block coding with imperfect channel estimation," in Proceedings of the IEEE International Conference on Communications (ICC'05), vol. 1, pp. 583-587, Seoul, Korea, May 2005.

[6] A. Stefanov and E. Erkip, "Cooperative space-time coding for wireless networks," IEEE Transactions on Communications, vol. 53, no. 11, pp. 1804-1809, 2005.

[7] P. A. Anghel, G. Leus, and M. Kaveh, "Distributed space-time cooperative systems with regenerative relays," IEEE Transactions on Wireless Communications, vol. 5, no. 11, pp. 31303141, 2006.

[8] J. Mietzner and P. A. Hoeher, "Improving the performance of mobile broadcasting systems using multiple base stations and distributed space-time codes," IET Communications, vol. 1, no. 3, pp. 348-353, 2007.

[9] J. Luo, R. S. Blum, L. J. Greenstein, L. J. Cimini Jr., and A. M. Haimovich, "Link-failure probabilities for practical cooperative relay networks," in Proceedings of the 61st IEEE Vehicular Technology Conference (VTC '05), vol. 3, pp. 1489-1493, Stockholm, Sweden, May-June 2005. 
[10] B. Sirkeci-Mergen and A. Scaglione, "Randomized space-time coding for distributed cooperative communication," IEEE Transactions on Signal Processing, vol. 55, no. 10, pp. 50035017, 2007.

[11] L. Zhang, L. J. Cimini Jr., L. Dai, and X.-G. Xia, "Relaying strategies for cooperative networks with minimal node communications," in Proceedings of the Military Communications Conference (MILCOM '06), pp. 1-7, Washington, DC, USA, October 2006.

[12] A. Nasipuri, J. Zhuang, and S. R. Das, "A multichannel CSMA MAC protocol for multihop wireless networks," in Proceedings of Wireless Communications and Networking Conference (WCNC'99), vol. 3, pp. 1402-1406, New Orleans, La, USA, September 1999.

[13] E. G. Larsson and P. Stoica, Space-Time Block Coding for Wireless Communications, Cambridge University Press, Cambridge, UK, 2003.

[14] J. Luo, R. S. Blum, L. J. Cimini Jr., L. J. Greenstein, and A. Haimovich, "Power allocation in a transmit diversity system with mean channel gain information," IEEE Communications Letters, vol. 9, no. 7, pp. 616-618, 2005.

[15] L. Zhang and L. J. Cimini Jr., "Efficient power allocation for decentralized distributed space-time block coding," submitted to IEEE Transactions on Wireless Communications, 2007.

[16] J. Luo, R. S. Blum, L. J. Cimini Jr., L. J. Greenstein, and A. M. Haimovich, "New approaches for cooperative use of multiple antennas in ad hoc wireless networks," in Proceedings of the 60th IEEE Vehicular Technology Conference (VTC '04), vol. 4, pp. 2769-2773, Los Angeles, Calif, USA, September 2004.

[17] S. Catreux, P. F. Driessen, and L. J. Greenstein, "Data throughputs using multiple-input multiple-output (MIMO) techniques in a noise-limited cellular environment," IEEE Transactions on Wireless Communications, vol. 1, no. 2, pp. 226-235, 2002.

[18] S. M. Alamouti, "A simple transmit diversity technique for wireless communications," IEEE Journal on Selected Areas in Communications, vol. 16, no. 8, pp. 1451-1458, 1998.

[19] L. Liu and H. Ge, "Space-time coding for wireless sensor networks with cooperative routing diversity," in Proceedings of the 38th Asilomar Conference on Signals, Systems and Computers (ACSSC '04), vol. 1, pp. 1271-1275, Pacific Grove, Calif, USA, November 2004.

[20] T. Miyano, H. Murata, and K. Araki, "Cooperative relaying scheme with space time code for multihop communications among single antenna terminals," in Proceedings of the IEEE Global Telecommunications Conference (GLOBECOM'04), vol. 6, pp. 3763-3767, Dallas, Tex, USA, November-December 2004.

[21] T. Miyano, H. Murata, and K. Araki, "Space time coded cooperative relaying technique for multihop communications," in Proceedings of the 60th IEEE Vehicular Technology Conference (VTC '04), vol. 7, pp. 5140-5144, Los Angeles, Calif, USA, September 2004.

[22] T. Koike, M. Tanaka, and S. Yoshida, "System-level evaluation of STC cooperative relaying scheme in multihop IVC networks," in Proceedings of the 2nd IEEE VTS Asia Pacific Wireless Communications Symposium (APWCS '05), pp. 6064, Hokkaido University, Japan, August 2005.

[23] A. D. Coso, S. Savazzi, U. Spagnolini, and C. Ibars, "Virtual MIMO channels in cooperative multi-hop wireless sensor networks," in Proceedings of the 40th Annual Conference on Information Sciences and Systems (CISS '06), pp. 75-80, Princeton, NJ, USA, March 2006. 